\title{
Gas breakdown mitigation in satellite slip rings
}

F. Avino, ${ }^{1}$ P. Martens, ${ }^{1}$ A. A. Howling, ${ }^{1}$ D. Bommottet, ${ }^{2}$ and I. Furno ${ }^{1}$

1) Ecole Polytechnique Fdrale de Lausanne (EPFL), Swiss Plasma Center (SPC), CH-1015 Lausanne,

Switzerland

${ }^{2)}$ RUAG Space, CH-1260 Nyon, Switzerland.

(Dated: 8 November 2018)

Direct current (DC) gas breakdown is experimentally investigated for high-voltage circular conductors and insulators that reproduce the main features of a satellite slip ring. Breakdown voltages are measured in the pressure range $10^{-3}-10$ mbar, where gas breakdown is predominant with respect to vacuum breakdown. The measured breakdown curves show clear similarities with Paschen's curve, which is generally associated with parallel plate electrodes. It is shown that the low-pressure branch of the measured curves is determined by breakdown between the high-voltage ring and the grounded vacuum chamber, whereas the high-pressure branch is due to discharges between the high-voltage ring and the adjacent grounded rings. A technical solution is introduced to inhibit the gas discharges at low-pressures in a slip ring assembly: The diameter of the grounded conducting discs is extended, strongly increasing the measured breakdown voltages by modifying the electric field distribution. The safe operating pressure range of the satellite slip ring is thereby increased by two orders of magnitude.

\section{INTRODUCTION}

Electrical breakdown in high-voltage (HV) systems can lead to severe damage of components., ${ }^{1,2}$ Breakdown in gases has been investigated via numerical ${ }^{3-5}$ and experimental ${ }^{6-9}$ studies, exploring a variety of configurations of increasing complexity, ${ }^{10}$ with different gases as insulating media. ${ }^{11,12}$ Preventing breakdown in electrical circuits of satellites is a technological challenge. Satellites experience a wide range of pressure, from atmospheric pressure before launch to high vacuum during the depressurization phase. Also, many poorly-defined parameters characterise the environment surrounding a satellite during its operating life: Pressure fluctuations could occur because of surface degassing, outgassing of trapped volumes, gas thruster exhaust, or micro-meteoroid impact, and low energy ions could back-flow from an ion thruster plume.

The satellite components that are susceptible to electrical breakdown are the solar panels, ${ }^{13-15}$ and the slip ring assembly (SRA), which is part of the power transmission circuit of a satellite. ${ }^{16}$ From actual bus voltages in the range $28-100 \mathrm{~V}$, evaluation of higher voltages in the range $300-600 \mathrm{~V}$ is under way, with the corresponding higher risk of electrical breakdown. These voltages could be required to power new generations of ion and Hall effect thrusters. ${ }^{17}$ Power efficiency could also be improved by reducing the operating currents in the power lines and the corresponding ohmic losses. Lower power system costs ( $\sim 30 \%$ reduction) and substantial mass savings $(\sim 50 \%$ reduction) could be achieved.

This work focuses on gas breakdown on the standard cylindrical configuration of a SRA, which transfers the electrical power from the rotating solar panels to the rest of the satellite via gold-plated brushes slipping on gold-plated rings. This geometry includes a stack of exposed biased conductors inside a conducting housing at the ground reference voltage of the satellite, as illustrated in Fig. 1. The aim here is to optimize the slip ring design to inhibit breakdown ${ }^{18}$ in the low pressure range. The baseline for our investigations is a previous work ${ }^{16}$ where the main features of gas breakdown in a simplified SRA are described by experiment and numerical simulation.

This paper is organised as follows: Sec. II presents the experimental setup. In Sec. III A, an initial electrode design is used to show that breakdown at low pressure occurs via the conducting housing. Based on this consideration, Sec. III B introduces a technical solution to inhibit SRA breakdown at low pressures. Sec. IV describes numerical simulations which qualitatively confirm the measured breakdown curves. The results are discussed in Sec. V before concluding in Sec. VI.

\section{EXPERIMENTAL SETUP AND PROCEDURE}

A schematic of the experimental setup is shown in Fig. 2. Measurements are performed using air inside a grounded cylindrical stainless steel vacuum chamber, $32 \mathrm{~cm}$ diameter and $40 \mathrm{~cm}$ high. A base pressure of $\sim 10^{-3}$ mbar can be attained using a rotary pump. The pressure is adjusted either by varying the pumping speed through a valve at the pump input, or by regulating the air flow rate with a valve on the vacuum chamber. Pressure measurements are performed with two baratrons (2mbar and 1000mbar range), a Pirani gauge and a cold cathode gauge.

Two different electrode designs are presented in Sec. III A and B respectively. In all cases, the $\mathrm{HV}$ electrode is biased by a Danbridge $30 \mathrm{kV}$ non-destructive insulation tester type JP30 A. ${ }^{20}$ Three current (1, 10 and 100 $\mu \mathrm{A})$ and voltage $(0-3,0-10$ and $0-30 \mathrm{kV}$ DC) ranges provide a sufficient flexibility for the operating parameters. For the measurements in Sec. III A, the output signals of current and voltage are measured by an oscilloscope. Breakdown triggers a fast drop and rise, respectively, of the voltage and current signals as shown in Fig. 3. Break- 


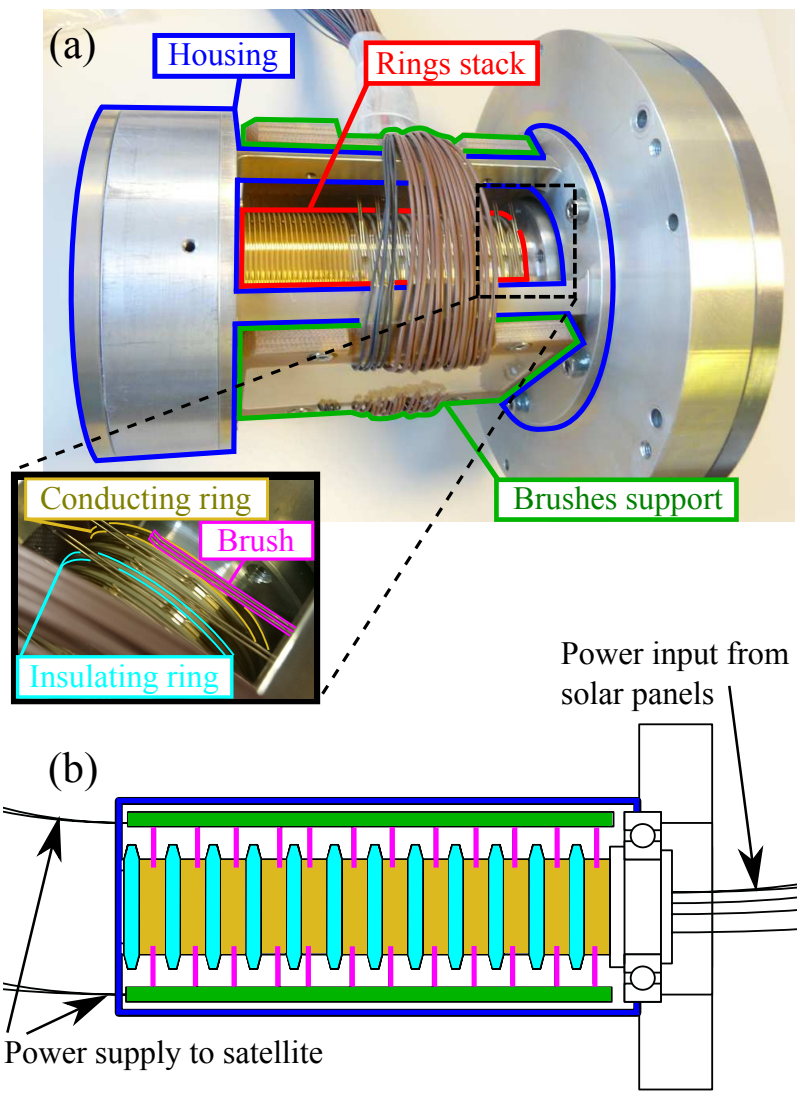

FIG. 1. (a) Photograph of a cylindrical slip ring. ${ }^{19}$ The conducting housing is partially removed to show the slip ring components such as brushes, and conducting and insulating rings. (b) Schematic of the slip ring with the colours of the components corresponding to the labels in (a).

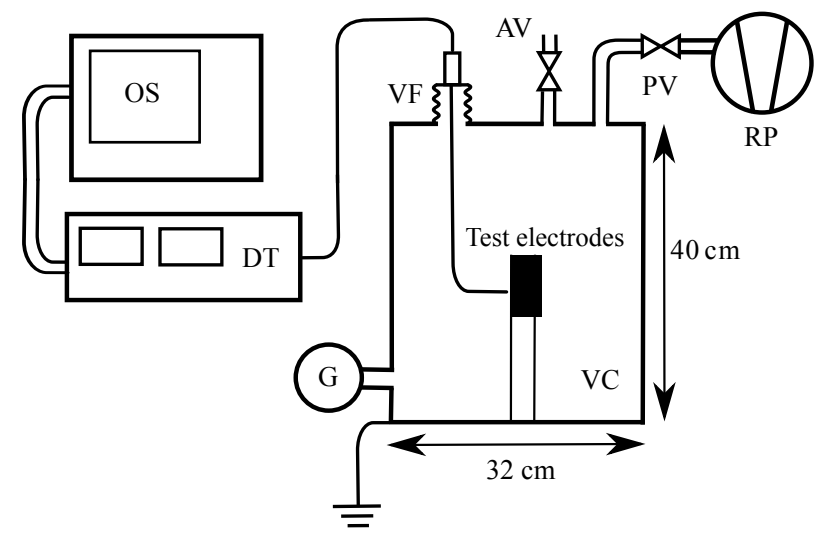

FIG. 2. Schematic of the experimental setup. The electrode designs are tested inside the cylindrical vacuum chamber (VC). The pumping system includes a rotary pump (RP), a valve (PV) to regulate the pumping speed, and a valve (AV) to set the air flow from outside the chamber. The pressure gauges $(\mathrm{G})$, the dielectric tester (DT) and the $\mathrm{HV}$ vacuum feed-through (VF) used to bias the HV electrode are shown. Measurements were acquired with an oscilloscope (OS).

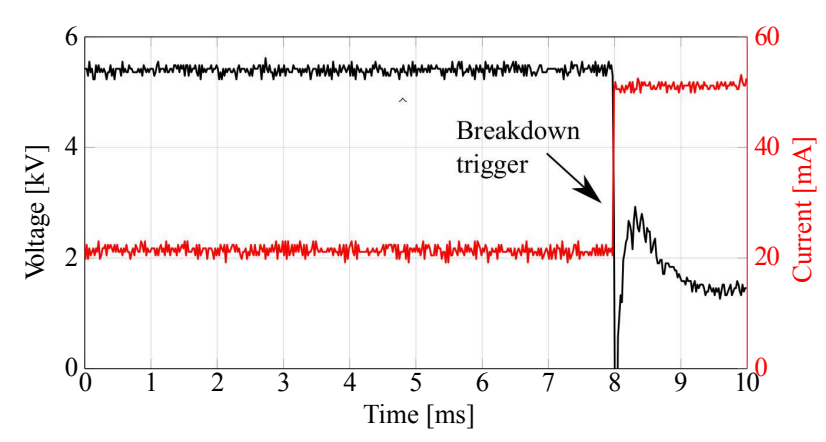

FIG. 3. Current and voltage signals measured on the oscilloscope.

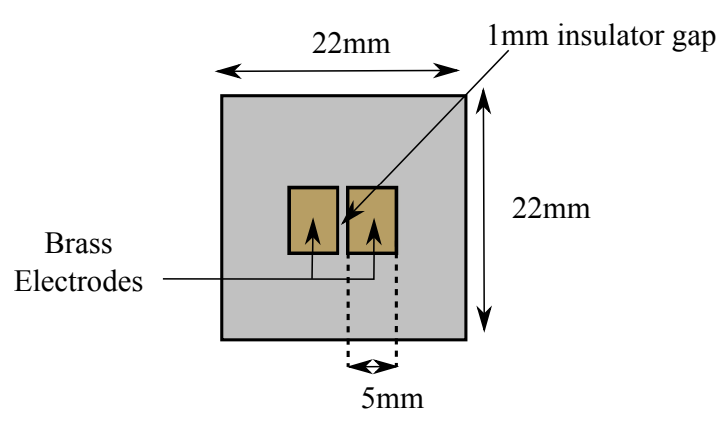

FIG. 4. Schematic of the first electrode design on a PVC substrate.

down curves are measured by adjusting the pressure to the required value, then increasing the voltage with an average ramp speed between 10 and $100 \mathrm{~V} / \mathrm{s}$ to avoid over-voltage effects. ${ }^{16}$ Once breakdown is detected, the procedure is repeated for the next pressure.

\section{EXPERIMENTAL MEASUREMENTS OF BREAKDOWN CURVES}

\section{A. Chamber wall influence: Initial tests with plane electrodes}

An initial test using a pair of plane electrodes was performed to study the gas discharge occurring between the HV electrode and the surrounding vacuum chamber. This feature was underlined in previous works, ${ }^{21-23}$ but is further explored in this section for its relevance to the breakdown mitigation technique presented in Sec. IIIB. A schematic of the electrodes in Fig. 4 shows two $5 \mathrm{~mm}$ square brass electrodes, separated by a $1 \mathrm{~mm}$ insulator gap. A positive high voltage was applied to one electrode (HV) by using the dielectric tester, while the other electrode (GE) was grounded, as shown for these electrodes in Fig. 5(a).

A first set of measurements was performed with this setup inside the vacuum chamber to obtain the reference curve of gas discharge shown with black circles in Fig. $5(\mathrm{~b})$. The error-bars are obtained from the standard de- 
viation of the measured breakdown values. The breakdown minimum voltage of $V_{B} \approx 420 \mathrm{~V}$ was measured at $\sim 1$ mbar.

A second set of measurements was then obtained by disconnecting the ground electrode, leaving it floating. Consequently, any electrical breakdown could only occur between the HV electrode and the walls of the chamber, as sketched in Fig. 5(a) with the magenta curve. The results are shown in Fig. 5(b) by magenta squares. It can be seen that the black reference curve is reproduced in the low pressure range, up to $2 \times 10^{-1}$ mbar. This confirms that at low pressures, the breakdown occurs preferentially on longer paths, i.e. between the HV electrode and the chamber wall. The absence of short paths at higher pressures, where the ionization process would have been more efficient, results in higher voltages with respect to the black reference curve.

A third set of measurements to explore the high pressure branch of the curve was made by inserting the electrodes inside a conducting structure, depicted in grey in Fig. 5(a). This structure could be closed and isolated from the rest of the chamber with the sliding cover sketched in magenta/dashed blue in Fig. 5(a), blocking the electrical paths from the HV electrode to the chamber wall. The resulting breakdown curve is shown by blue diamonds in Fig. 5(b). The complementary superposition with the previous curves is observed, reproducing the breakdown voltages in the high-pressure range, down to $5 \times 10^{-1}$ mbar. The absence of long paths at lower pressures, where the ionization process would have been more efficient, results in higher voltages with respect to the black reference curve.

Hence, the black reference curve in Fig. 5(b) is shown to be composed of two distinct sections: Breakdown to the walls for low pressure, and breakdown between adjacent electrodes at high pressure.

\section{B. Gas discharge mitigation in a SRA at low pressures: Second experiment with a stack of ring electrodes}

This section presents a technical solution to inhibit gas discharge breakdown between a SRA and the surrounding walls, for low pressures. A second electrode design which reproduces the main features of a standard SRA is used, ${ }^{16}$ as shown in Fig. 6. The stack of rings consists of a central brass HV-ring, $1 \mathrm{~mm}$ thick, with an internal diameter of $34 \mathrm{~mm}$ and an external diameter of 38 $\mathrm{mm}$. This is held between two adjacent insulating rings of the same dimensions, made of Vetronite G-11, having a dielectric strength of $20 \mathrm{kV} / \mathrm{mm} .{ }^{24}$ Two $0.2 \mathrm{~mm}$ thick grounded brass discs enclose the assembly, which is held in the middle of the vacuum chamber by a Teflon support (Fig. 2). The central HV-ring is positively biased with respect to the two external grounded discs.

Breakdown measurements were performed without the grounded discs, and then for successively wider diameter of the discs. The chosen diameters were $38 \mathrm{~mm}, 40 \mathrm{~mm}$,
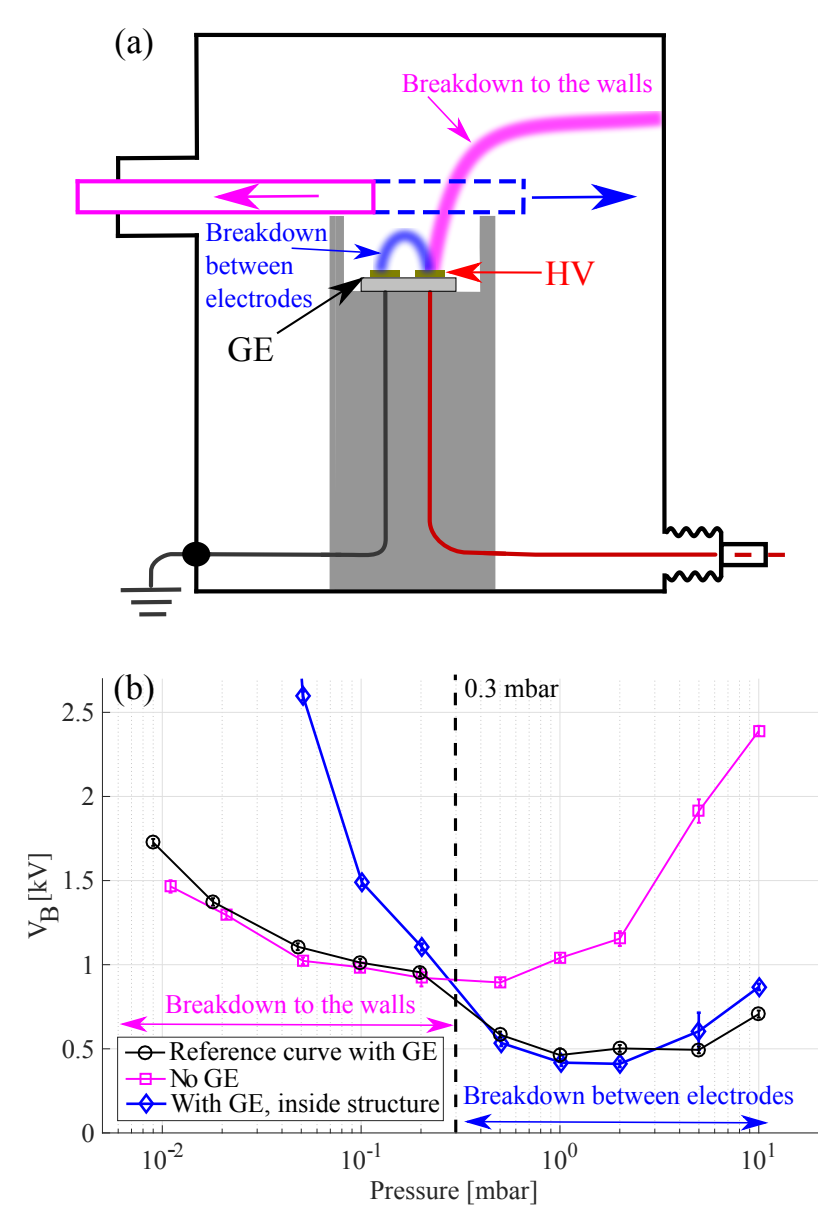

FIG. 5. (a) Schematic of the setup with the grounded (GE) and high-voltage (HV) electrodes of Fig. 4. Breakdown between the $\mathrm{HV}$ electrode and the walls is schematically represented by the magenta curve; breakdown between the electrodes is sketched by the blue curve. A sliding cover can be closed to prevent breakdown to the walls. (b) Black circles indicate the breakdown reference curve for the electrodes (Fig. 4 ) in the vacuum chamber. Magenta squares mark measurements when the grounded electrode was disconnected. Blue diamonds are for when discharges were only possible between the two electrodes.

$44 \mathrm{~mm}, 48 \mathrm{~mm}$ and $58 \mathrm{~mm}$. These diameters correspond to two conducting boundaries extending beyond the $\mathrm{HV}$ ring radius by $+0 \mathrm{~mm},+1 \mathrm{~mm},+3 \mathrm{~mm},+5 \mathrm{~mm}$, and +10 $\mathrm{mm}$, respectively. The breakdown curve measurements in the range $p=5 \times 10^{-3}-10$ mbar are presented in Fig. 7. A maximum bias of $6 \mathrm{kV}$ was applied to the HV-ring. The error bars correspond to the resolution of the voltmeter.

Comparing the breakdown curve of the $+0 \mathrm{~mm}$ discs with the curve for no discs reveals opposite trends either side of their crossing point (at $p \simeq 5 \times 10^{-1} \mathrm{mbar}$ ), similarly to the magenta and blue curves of Fig. 5(b). The presence of wider grounded discs enclosing the central HV-ring significantly increases the voltage for which the gas discharge occurs in the range $p=5 \times 10^{-3}-$ 


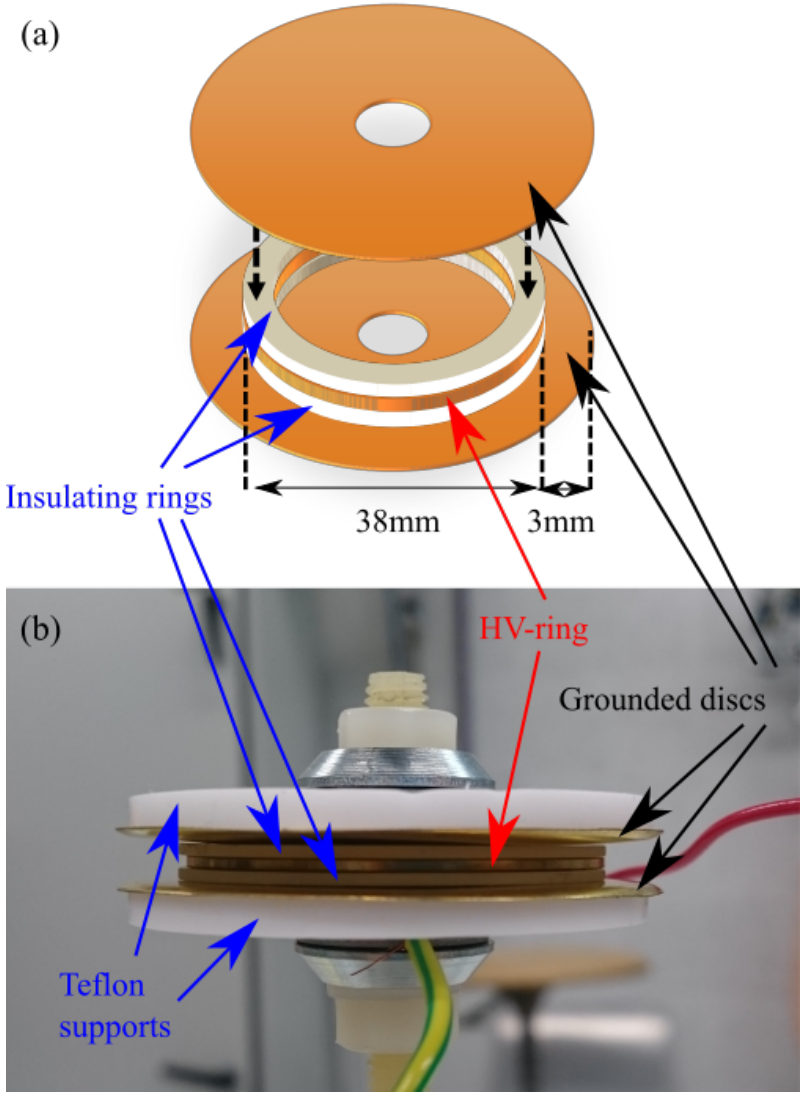

FIG. 6. (a) Schematic of the second setup of electrodes. The central HV-ring is isolated from the external grounded discs by two insulating rings (shown in white). The top disc is raised to show the lower part of the stack. The insulating support filling the internal volume inside the rings is not shown. (b) Photograph of the assembly.

$5 \times 10^{-1} \mathrm{mbar}$, in Fig. 7 . For an intermediate pressure of $10^{-1}$ mbar, the presence of grounded limiting discs $3 \mathrm{~mm}$ wider with respect to the central ring surface increases the breakdown voltage from $\approx 1 \mathrm{kV}$ to $\approx 4 \mathrm{kV}$. We can see that for the $+10 \mathrm{~mm}$ curve, gas breakdown is almost completely suppressed up to approximately $2 \times 10^{-1} \mathrm{mbar}$, for applied voltages below $6 \mathrm{kV}$. This is a significant improvement compared to the standard slip-ring where all rings have the same diameter (+0 mm curve in Fig. 7).

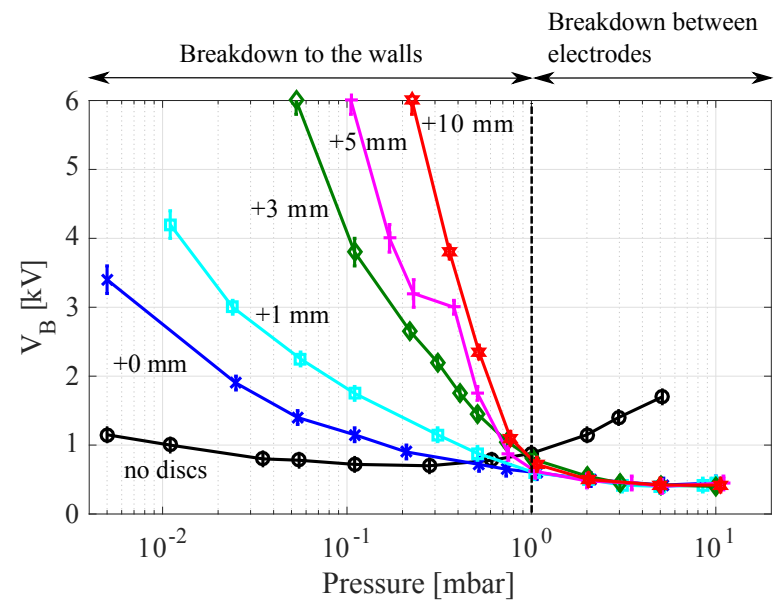

FIG. 7. Breakdown voltage as a function of the gas pressure for different grounded discs. The black circles are for the HV-ring alone inside the vacuum chamber. The other measurements refer to the assembly with grounded limiting rings of increasing diameter.

\section{NUMERICAL SIMULATIONS}

To further investigate the experimental results obtained in Sec. III B, numerical studies were performed with the finite element software COMSOL $4.2 .{ }^{25} \mathrm{~A}$ twodimensional axisymmetric model was used as shown in Fig. 8 with a vertical axis of symmetry at $r=0 \mathrm{~cm}$. Air at different pressures is set as the medium inside a 20 cm-diameter cylindrical grounded chamber. A simplified model of the SRA is placed in the middle of the chamber at $z=0 \mathrm{~mm}$. The central HV-ring is separated from the grounded discs by insulating rings. The grounded rings of different sizes are indicated with different colours, from those with the same dimension of the HV-ring $(+0 \mathrm{~mm}$, in red), up to the discs representing $+10 \mathrm{~mm}$ grounded limiting surfaces, indicated in yellow.

\section{A. Vacuum electric field distribution}

The vacuum electric field $\vec{E}=-\vec{\nabla} V$ is calculated from the Laplace equation $\nabla^{2} V=0$ using the appropriate voltage boundary conditions. Space charge effects and surface charge accumulation on insulators are neglected, given the low densities of electrons and ions before breakdown. In Figs. 9(a) and (b), the electric field intensity is illustrated with $1 \mathrm{kV}$ bias on the HV-ring, for the configuration with $+0 \mathrm{~mm}$ and $+10 \mathrm{~mm}$ discs, respectively. Detailed pictures of the SRA are shown in the corresponding insets. The electric field distribution is significantly modified by the wider grounded disc: In most of the vacuum chamber volume, the $E$-field decreases by three orders of magnitude. Moreover, a significant fraction of the electric field streamlines leaving the HV-ring surface (in black) reach the wall chamber for the $+0 \mathrm{~mm}$ 


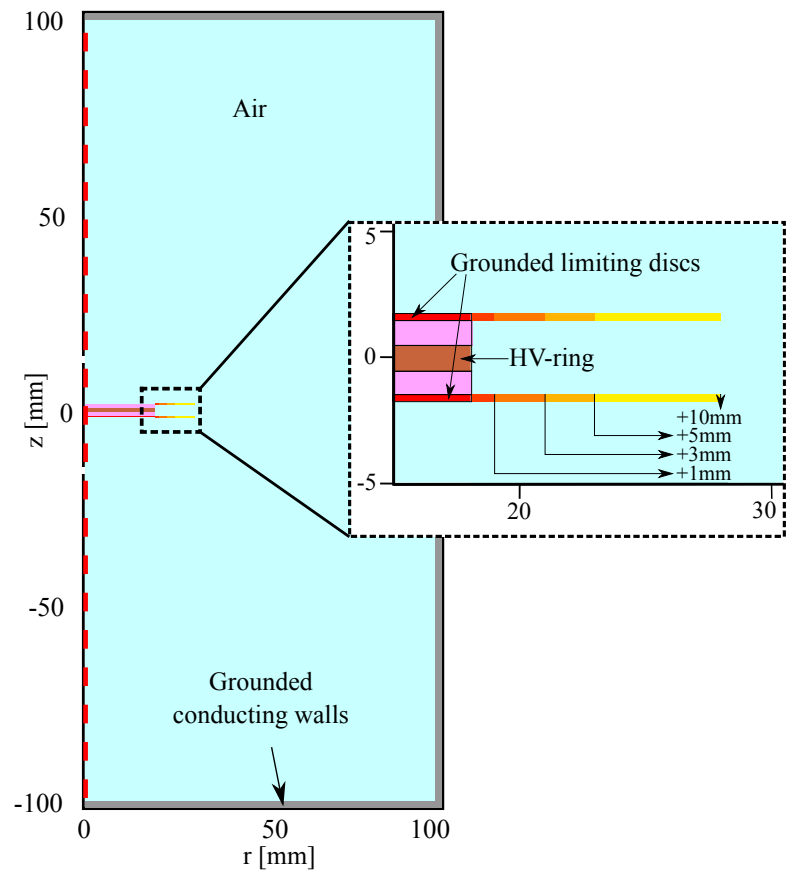

FIG. 8. Schematic of the simulation geometry. The vertical dashed red line corresponds to the axis of symmetry. The vacuum chamber walls are shown in grey. A detailed view of the SRA is also depicted: The HV-ring is shown in orange, the insulating components in magenta, and the grounded limiting discs in red, with the increasing diameters indicated with different colours up to yellow.

setup (a). On the contrary, the field lines are confined to the space between the discs for the $+10 \mathrm{~mm}$ configuration (b). A complementary view is given by the electric field streamlines leaving the chamber wall surface, indicated in grey. For the $+10 \mathrm{~mm}$ discs, these lines can reach the HV-ring surface only in a narrow central region.

The calculated field distribution is therefore consistent with the observation that breakdown is progressively suppressed on long electric field paths for increasing disc size: The grounded limiting discs are acting as partial Faraday screens for the HV ring.

\section{B. Gas breakdown in air}

Fluid simulations using a drift-diffusion model $^{26}$ for this SRA geometry ${ }^{16,23}$ were performed to complement the experimental measurements of electrical breakdown. The low-pressure range was explored, increasing the diameter of the grounded limiting discs from $+0 \mathrm{~mm}$ to $+3 \mathrm{~mm}$. The results shown in Fig. 10 show qualitative agreement with the measured breakdown curves in Fig. 7. The curves steepen in the low pressure range for increasing disc diameter, confirming the inhibition of the electrical breakdown towards the walls. The discrepancy compared to the experimental values could be due, for example, to the assumed values used for $\alpha$ and $\gamma$ in the numerical simulation. ${ }^{16,23}$

\section{DISCUSSION}

Intuitively, the breakdown voltage between electrodes in vacuum can be avoided by increasing the interelectrode gap. In contrast, gas breakdown occurs along the electric field path where the ionizing avalanche process is the most efficient, which will preferentially be along long paths at low pressures. This is relevant in complex geometries like in a SRA, where the available breakdown paths go from the millimeter scale between adjacent rings, up to tens of centimetres between a HV ring and the surrounding conducting housing. For lowenough pressures, gas discharge between the HV ring and the surrounding walls determines the breakdown voltage, as verified in Sec. III A.

In Sec. III B, experiments on a simplified stack of rings with the dimensions of a real SRA, showed that large-diameter grounded discs modify the low-pressure branch, increasing the breakdown voltage. This effect is related to the modified electric field distribution which inhibits the discharge on long electric field paths towards the walls, as shown by the numerical simulations presented in Sec. IV. The presence of grounded discs does not modify the high pressure branch of the breakdown curve, because the small distance between adjacent rings remains unchanged.

\section{CONCLUSIONS}

Gas breakdown has been investigated in the complex geometry of a Slip Ring Assembly (SRA). Electrical breakdown between the high-voltage electrode and the conducting housing dominates the low pressure branch of the measured breakdown curve. An innovative passive technical solution to inhibit gas breakdown at low pressures was to increase the diameter of the grounded rings, which then act as a partial Faraday screen of the HV ring, strongly reducing the electric field intensity towards the wall. The proximity of the grounded limiting discs is counter-intuitive from the point of view of vacuum breakdown, but this approach effectively inhibits gas breakdown which occurs at voltages much lower than for vacuum breakdown. The safe operating pressure range of the satellite slip ring is thereby increased by two orders of magnitude.

\section{ACKNOWLEDGMENTS}

This work was part of the High Voltage - Electrical Power System Architecture (HV-EPSA) project, and received funding from the SEFRI under grant agreement number 15.0340, in the framework of the European Union's Horizon 2020 research and innovation pro- 
(a)

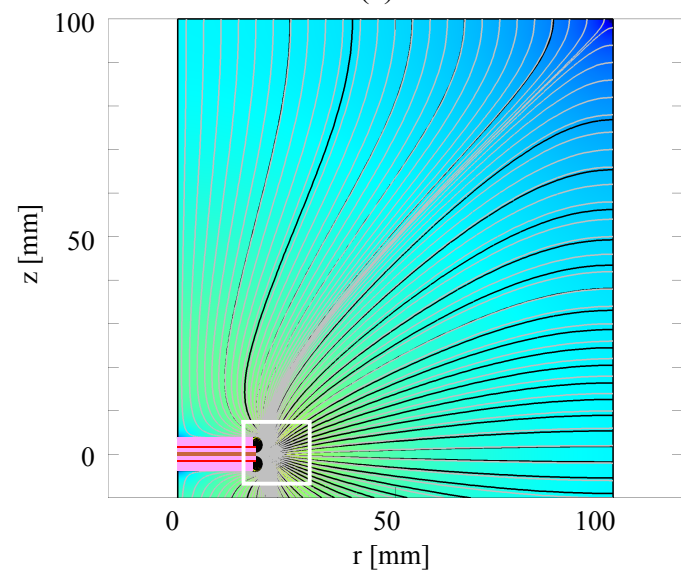

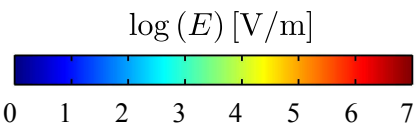

5
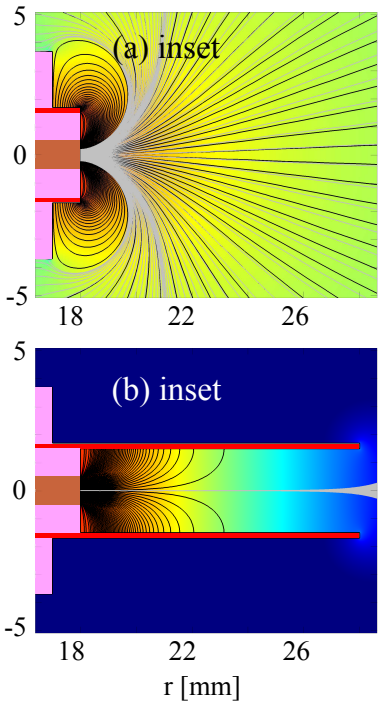

(b)

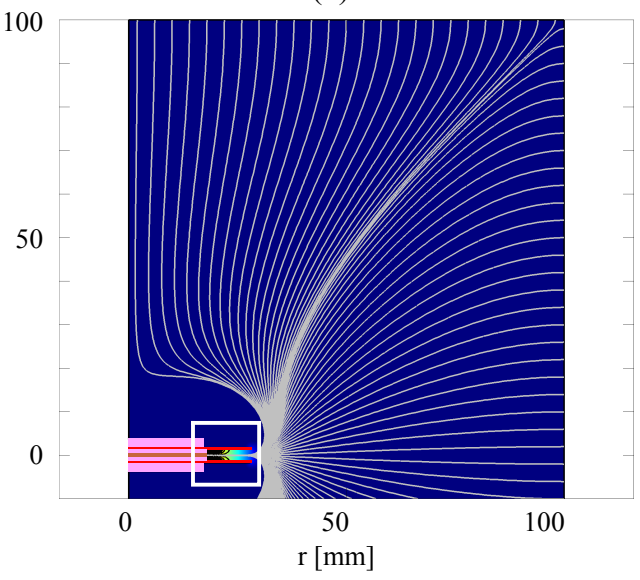

FIG. 9. Vacuum electric field distribution for (a) the $+0 \mathrm{~mm}$, and (b) the $+10 \mathrm{~mm}$ disc configurations. Detailed views of the slip ring assemblies are illustrated in the corresponding insets for (a) and (b) as marked by the white rectangles. The calculated electric field streamlines leaving the HV-ring are in black, and the streamlines leaving the vacuum chamber wall are in grey.

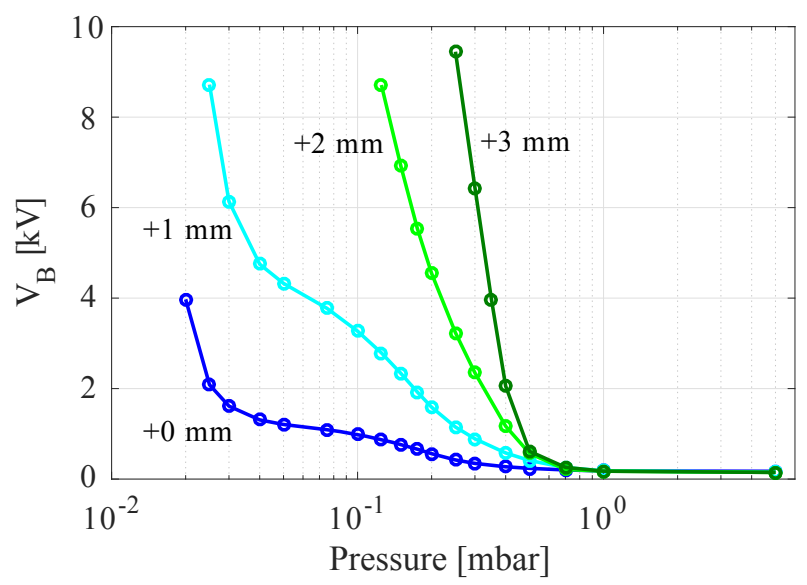

FIG. 10. Numerical simulations of breakdown voltage as a function of pressure, for several diameters of the grounded limiting discs.

gramme. The views and opinions expressed herein do not necessarily reflect those of the European Commission.

${ }^{1}$ Y.P. Raizer, Gas Discharge Physics, 1991, Springer.

${ }^{2}$ M.A. Lieberman, and A.J. Lichtenberg, Principles of Plasma Discharges and Material Processing, 2nd edition, 1994, New York Wiley.

${ }^{3}$ G.J.M. Hagelaar, F.J. de Hoog, and G.M.W. Kroesen, Phys. Rev. E 62, 1452 (2000).

${ }^{4}$ G.E. Georghiou, A.P. Papadakis, R. Morrow, and A.C. Metaxas J. Phys. D: Appl. Phys. 38, R303 (2005).

${ }^{5}$ W.H. Koh, and I.-H. Park, Vacuum 84, 550 (2010).
${ }^{6}$ P. Osmokrovic, M. Vujisic, K. Stankovic, A. Vasic, and B. Loncar, Plasma Sources Sci. Technol. 16, 643 (2007).

${ }^{7}$ Z.Lj. Petrović, N. Skoro, D. Marić, C.M.O. Mahony, P.D. Maguire, M. Radmilović, and G. Malović, J. Phys. D: Appl. Phys. 41, 194002 (2008).

${ }^{8}$ V.A. Lisovskiy, V.A. Koval, and V.D. Yegorenkov, Phys. Lett. A 375, 1986 (2011).

${ }^{9}$ V.A. Lisovskiy, R.O. Osmayev, A.V. Gapon, S.V. Dudin, I.S. Lesnik, and V.D. Yegorenkov, Vacuum 145, 19 (2017).

${ }^{10}$ L.A. Maglaras, and A.L. Maglaras, Transactions on Power Systems 3, 103 (2008).

${ }^{11}$ P. Osmokrovic, I. Krivokapić, and S. Krstić, IEEE Transactions on Dielectrics and Electrical Insulation 1, 77 (1994).

${ }^{12}$ B. Loncar, R. Radoslavljevic, M. Vujisic, K. Stankovic, and P. Osmokrovic, Acta Physica Polonica A, 119, 364 (2011).

${ }^{13}$ D.E. Parks, G.A. Jongeward, I. Katz, and V.A. Davis, Journal of Spacecraft and Rockets, 24, 367 (1987).

${ }^{14}$ D. E. Hastings, G. Weyl, and D. Kaufman, Journal of Spacecraft and Rockets, 27, 539 (1990).

${ }^{15}$ G. Bonin, N. Orr, R.E. Zee, and J. Cain, Solar Array Arcing Mitigation for Polar Low-Earth Orbit Spacecraft, 24th Annual AIAA/USU Conference on Small Satellites (2010).

${ }^{16}$ R. Schnyder, A.A. Howling, D. Bommottet, and C. Hollenstein, J. Phys. D: Appl. Phys. 46, 285205 (2013).

${ }^{17}$ A. Leporini, V. Giannetti, T. Andreussi, D. Pedrini, A. Rossodivita, A. Piragino, M. Andrenucci, and D. Estublier, Development of a $20 \mathrm{~kW}$-class Hall effect thruster, Proceedings of Space Propulsion, (2016).

${ }^{18}$ T. Yoke, S. Iwai, A.R. Khan, H. Masui, M. Iwata, K. Toyoda, and M. Cho, IEEE Trans. Plasma Science 41, 3477 (2013).

${ }^{19}$ RUAG Space Switzerland Nyon (RSSN), slip ring.

${ }^{20} \mathrm{http}: / /$ www.danbridge.com/files/jp30a_manual_jan2011.pdf.

${ }^{21}$ N. Spyrou, R. Peyrous, N. Soulem, and B. Held, J. Phys. D: Appl. Phys. 28, 701 (1995).

${ }^{22}$ D. Marić, N. Skoro, P.D. Maguire, C.M.O. Mahony, G. Malović, and Z.Lj. Petrović, Plasma Sources Sci. Technol. 21, 035016 (2012). 
${ }^{23} \mathrm{R}$. Schnyder, DC Breakdown in gases for complex geometries from high vacuum to atmospheric pressure, EPFL Lausanne, Switzerland, $\mathrm{PhD}$ thesis $\mathrm{n}^{\circ} 5962$ (2013).
${ }^{24}$ Von Roll Holding AG, Vetronite G-11.

${ }^{25}$ COMSOL Inc. and www.comsol.com.

${ }^{26}$ N. Leoni, and B. Paradkar, Proc. IS\&T's NIP (Springfield, VA), p229 (2009). 